\title{
Petits ARN nucléaires et connectivites
}

Les petits ARN nucléaires sont impliqués dans la maturation des ARN messagers. Des anticorps qui reconnaissent ces petits ARN et leurs protéines associées sont des marqueurs précieux de nombreuses connectivites.

\section{Jean-Pierre Liautard Chargé de recherches à l'Inserm}

\section{Marcel Rucheton \\ Chargé de recherches à l'Inserm}

\section{Hubert Graafland \\ Assistant des hôpitaux. Assistant à la faculté de médecine de Montpellier.}

\section{RÉFÉRENCES}

1. Tan EM. Autoantibodies to nuclear antigens (ANA): their immunobiology and medicine. $A d v$ Immunol 1982; 33: $167-240$.

2. Lemer MR, Steitz JA. Antibodies to small nuclear RNAs complexed with proteins are produced by patients with systemic lupus erythematosus. Proc Natl Acad Sci USA 1979; 76: 549599.

3. Busch H, Reddy R, Rothblum L, Choi YE. SnRNA, SnRNPs and RNA processing. Ann Rev Biochem I 982; 5 I.

4.Zieve G. Two groups of small stable RNAs. Cell 1981; 25: 296-7.

\section{ADRESSE}

J.P. Liautard : Inserm U. 249 et centre de recherche de biologie moléculaire du Cnrs. Route de Mende, B.P. 5051, 34033 Montpellier cedex.

M. Rucheton : Inserm $U$ 236, centre Paul Lamarque. Hôpital Saint-Êloi, B.P. 5054, 34033 Montpellier cedex.

H. Graafland : Institut d'hématologie. Centre de transfusion sanguine, a venue Émile-Jeanbrau, B.P. 1213,340 I 0 Montpellier cedex. es découvertes récentes sur la structure et la fonction des antigènes nucléaires impliqués dans certaines maladies autoimmunes ont permis de mieux comprendre un des mécanismes fondamentaux de la biologie : la maturation des ARN messagers. Ces découvertes, quoiqu'utilisant les méthodes de la biologie moléculaire moderne, sont étroitement tributaires de l'emploi des anticorps autoimmuns. En contrepartie, les travaux sur la nature des antigènes reconnus permettent une meilleure nosologie des connectivites et la formulation de quelques hypothèses nouvelles sur l'étiologie de ces maladies, qui pourraient être fécondes dans un avenir proche.

Après un bref historique, nous ferons le point sur l'état actuel des connaissances concernant les ribonucléoprotéines nucléaires que reconnaissent les anticorps autoimmuns. Nous décrirons ensuite les nouvelles techniques qui devraient bientôt être utilisées pour le diagnostic. Enfin, à la lumière des résultats, nous tenterons d'étayer une hypothèse virale de l'étiologie des connectivites.

Les anticorps capables de réagir contre les constituants nucléaires solubles en milieu salin dits antiENA (pour Extractable Nuclear Antigens) sont connus depuis la description de Holmann et coll. en 1959 , néanmoins la première implication clinique doit être attribuée à Tan et Kunkel qui ont décrit en 1966 la présence spécifique d'anticorps nommés anti-Sm dans le lupus érythémateux disséminé
(LED). En 1971, Mattioli et Reichlin décrivent une spécificité dite anti-Mo qui prendra avec Sharp et coll. en 1971 le nom d'anti-RNP (pour ribonucléoprotéine) et sera associée à une nouvelle entité clinique, la connectivite mixte (CM).

Par la suite, d'autres autoanticorps reconnaissant des antigènes nucléaires solubles furent progressivement décrits; leur liste, non exhaustive, est donnée dans le tableau I qui résume aussi les principales implications cliniques de ces anticorps.

\section{Une aide au diagnostic}

C'est probablement pour l'anticorps anti-Sm que la spécificité diagnostique est la meilleure; sa présence fait d'ailleurs maintenant partie des critères biologiques officiels de l'American Rheumatic Association pour le diagnostic du LED et il est à prévoir que l'amélioration de la sensibilité des méthodes de détection le placera au même rang que les anticorps anti-ADN bicaténaires.

Les anticorps anti-RNP, en revanche, ne sont pas des marqueurs absolument spécifiques des connectivites mixtes. Cette maladie, discutée sur le plan nosologique, de diagnostic différentiel souvent très difficile notamment avec la sclérodermie ou la polymyosite, pose pourtant un problème crucial pour le médecin traitant puisqu'il semble probable qu'une connectivite mixte serait sensible à une corticothérapie alors que les deux autres maladies seraient plus résistantes [1].

Enfin la présence d'anticorps anti- 
Ro (SS-A) ou anti-La (SS-B) peut aider au diagnostic et au pronostic du syndrome sec (salivaire ou lacrymal) et éventuellement aux troubles congénitaux de la conduction cardiaque chez les nouveaux-nés des mères présentant des autoanticorps de spécificité Ro.

\section{Nature moléculaire des petits ARN}

\section{Antigènes RNP et $\mathrm{Sm}$}

De nombreux travaux préliminaires avaient été réalisés pour essayer de définir la nature et la structure des antigènes Sm, RNP ou La [1] (tableau II). Mais ce sont les travaux de Leiner et Steitz [2] qui permirent la première identification de ces antigènes. Ils utilisèrent la propriété qu'a la protéine $A$ du staphylocoque de s'accrocher aux anticorps pour purifier les complexes antigènesanticorps. Ils montrèrent ainsi que l'antigène RNP est en fait la particule ribonucléoprotéique contenant le petit ARN nucléaire $U_{1}$ (snRNP $U_{1}$ ) tandis que l'antigène $\mathrm{Sm}$ correspond à 5 particules (snRNP) contenant les petits ARN nucléaires $U_{1}, U_{2}, U_{4}, U_{5}$ et $U_{6}[2$, 3]. Ces petits $A R N$ sont exclusivement nucléaires et relativements abondants $\left(10^{6}\right.$ molécules environ pour le snARN $U_{1}$ ). Leur séquence primaire et leur structure secondaire ont été déterminées [3, 4].

\begin{tabular}{|c|c|c|c|}
\hline RELAT & $\begin{array}{l}\text { Tableau } \\
\text { N ENTRE LES ANTIGENES } \\
\text { réalisé d'après }\end{array}$ & $\begin{array}{l}\text { T LE SYNDR } \\
\text { an [1] }\end{array}$ & ME CLINIQUE \\
\hline antigène & association clinique & références & $\begin{array}{c}\text { spécificité } \\
\text { diagnostique }\end{array}$ \\
\hline RNP & $\begin{array}{l}\text { lupus érythémateux dissé- } \\
\text { miné (LED) (30 à } 40 \%) \\
\text { connectivite mixte (cm) } \\
\text { (95 à } 100 \%) \\
\text { LED }(50 \%) \\
\text { GS }(60-70 \%)\end{array}$ & $\begin{array}{l}{[1,9]} \\
{[2,3,9]}\end{array}$ & $\begin{array}{l}\text { très bonne } \\
\text { bonne, se trouve } \\
\text { aussi dans : scléro- } \\
\text { dermie, Goujerot- } \\
\text { Sjogren (GS). } \\
\text { lupus discoïde }\end{array}$ \\
\hline SS-A/Ro & $\begin{array}{l}\text { LED ( }(30-40 \%) \\
\text { lupus néonatal }\end{array}$ & {$[4,6,9]$} & $?$ \\
\hline SS-B/La & $\begin{array}{l}\text { GS }(50-60 \%) \\
\text { LED }(10-15 \%)\end{array}$ & {$[5,6,7,9]$} & $?$ \\
\hline Scl 70 & sclérodermie (15-20\%) & [9] & très bonne \\
\hline $\begin{array}{l}\text { anti- } \\
\text { centromère }\end{array}$ & *CREST(70-90\%) & [9] & \\
\hline Ma & - LED (20\%) & [9] & $?$ \\
\hline $\begin{array}{l}\text { PCMA } \\
\text { PM }\end{array}$ & $\begin{array}{l}\text { LED }(5: 10 \%) \\
\text { syndrome de chevauche- } \\
\text { ment polymyosite-scléro- } \\
\text { dermie }(87 \%) \text { dermato } \\
\text { myosite }(17 \%)\end{array}$ & $\begin{array}{l}\text { [9] } \\
{[9]}\end{array}$ & ? \\
\hline $\mathrm{Mi}-1$ & dermato myosite (11\%) & [9] & $?$ \\
\hline Jo 1 & polymyosite (31\%) & [8] & ? \\
\hline
\end{tabular}

* CREST : calcinose, syndrome de Raynaud, dysfonction oesophagienne, sclérodactylie, telangiectasie.

\begin{tabular}{|c|c|c|c|c|c|c|}
\hline \multicolumn{7}{|c|}{$\begin{array}{c}\text { Tableau II } \\
\text { TABLEAU RĖCAPITULATIF DE LA STRUCTURE DE LA FONCTION DES ANTIGÉNES }\end{array}$} \\
\hline antigène & RNP & nomenclature & $\begin{array}{l}\text { ARN } \\
\text { longueur }\end{array}$ & extrémité $5^{\circ}$ & $\begin{array}{c}\text { protéines } \\
\text { antigéniques }\end{array}$ & $\begin{array}{c}\text { rôle } \\
\text { proposé }\end{array}$ \\
\hline $\mathrm{Sm}$ & snRNP & $U_{1} U_{2} U_{4}$ & $106-198$ & $m^{3} G p p p$ & $13 K 26 K 27 K$ & maturation des pre-mRNA \\
\hline RNP & snRNPU, & $U_{1}$ & 165 & $m^{3} G p p p$ & $68 K 33 K 22 K$ & épissage des pre-mRNA \\
\hline Ro & RoRNP & $Y_{1} Y_{2} Y_{3} Y_{1}$ & $90-110$ & pppG & $\begin{array}{c}90 \mathrm{~K} \text { et } 94 \mathrm{~K} \\
\text { ou } 60 \mathrm{~K}\end{array}$ & $?$ \\
\hline La & LaRNP & $\begin{array}{c}V_{A_{1}} V A_{\text {II }} \\
E B E R_{1} \text { EBER } \\
Y_{1}-Y_{4}-\text { preSS } \\
\text { pre-tRNA }\end{array}$ & $80-173$ & $\operatorname{ppp}_{\mathrm{G}}$ & $50 K$ & facteur de transcription \\
\hline jo-1 & - & tRNA $^{\text {his }}$ & 75 & $p G$ & $64 K$ & AAtRNA synthetase \\
\hline
\end{tabular}




\section{REFEERENCES}

5. Brunel C, Sri-Widada J, Lelay MN, Jeanteur $\mathrm{P}$, Liautard JP. Purification and characterization of a simple ribonucleoprotein particle containing small nucleoplasmic RNAs (snRNP) as a subse of RNP containing heterogeneous nuclear RNA (hnRNP) from HeLa cells. Nucleic Acids Res I 981;9:8 I 5-30.

6. Assens C, Liautard JP, Sri-Widada J, Brune C, Jeanteur $P$. Highly purified snRNPs retain antigenic determinants towards anti $\mathrm{Sm}$ antibodies. Biochem Biophys Res Commun 1 982; 106: 953-60.

7. Liautard JP, Sri-Widada J, Brunel C, Jeanteur P. Structural organization of ribonucleoproteins containing small nuclear RNAs from HeLa cells. 7 Mol Biol 1982; 162: 623-43.

8. Pettersson I, Hinterberger $M$, Mimori $T$, Gottlieb E, Steitz JA. The structure of mammalian small nuclear ribonucleoprotcins. Identification of multiple protein components reactive with anti- $\left(\mathrm{U}_{1}\right)$ ribonucleoprotein and anti-Sm autoantibodies. 7 Biol Chem 1984; 259: 5907-I4.

9. Billings PB, Allen RW, Jensen FC, Hoch SO Anti-RNP monoclonal antibodies derived from a mouse strain with Lupus-like autoimmunity. $\mathcal{f}$ Immunol I 982; 1 28: 1 I 76-80.

I0. Sri-Wadada J, Liautard JP, Assens C, Brunel C. Primary structure identification of snRNAs present in highly purified snRNPs from HeLa cells. Mol Biol Rep 198 r ; 8: 29-36.

I I. Long BH, Schrier WH. Isolation from Friend erythroleukemia cells of an RNAse-sensitive nuclear matrix fibril fraction containing hnRNA and snRNA. Biol Cell 1 983; 48: 99-108.

12. Lerner MR, Steitz JA. Snurps and Scyrps. Cell I98I; 25: 298-300.

13. Wollin SL, Steitz JA. Genes for two small cytoplasmic Ro RNA are adjacent and appear to be single copy in the human genome. Cell 1983 ; 32:735-44.

14. Hendrick JP, Wolin SL, Rinke J, Lerner MR, Steitz JA. Ro small cytoplasmic ribonucleoproteins are a subclass of $\mathrm{La}$ ribonucleoproteins: further characterization of the Ro and La small ribonucleoproteins from uninfected mammalian cells. Mol Cell Biol I 981 ; 1 : 1 1 38-49.

I 5. Francour AM, Mathews MB. Interaction between VA RNA and the lupus antigen La : formation of a ribonucleoprotein particle in vitro. Proc Natl Acad Sci USA 1982; 79: 6772-6.

16. Steitz JA, Wolin SL, Rinke J, et al. Small ribonucleoproteins from eukaryotes: structures and roles in RNA biogenesis. Cold Spring Harbor Symp Quant Biol 1983; 47: 893-900.

17. Stefano JE. Purified lupus antigen La recognizes an oligouridylate stretch common to the 3 , termini of RNA polymerase III transcripts. Cell 1984;36: 1 45-54

18. Mathews MB, Bernstein RM. Myositis autoantibody inhibits histidine-tRNA synthetase: a model for autoimmunity. Nature 1983; 304:

Le fait que l'anti $\mathrm{Sm}$ reconnaisse les cinq particules implique qu'elles contiennent un antigène commun. De plus, si on prépare les snRNP à haute force ionique, on obtient des particules résiduelles qui possèdent chacune un ensemble de protéines identiques [5] (leur poids moléculaire est compris entre 12 et $29 \mathrm{~K}$ daltons) et qui ont conservé une réactivité avec l'anti-Sm, mais perdu les déterminants antigéniques RNP [6]. Les séquences d'ARN que reconnaissent ces protéines sont en simple brin et contiennent la séquence nucléotidique $A(U)_{n} G[7]$.

Les snRNA ne sont pas antigéniques par eux-mêmes. Ce sont les protéines associées qui conferent l'antigénicité aux snRNP (figure 1). L'utilisation de la méthode de transfert des protéines sur nitrate de cellulose puis de détection des protéines antigéniques directement sur le filtre (immunoblotting) a permis de préciser quelles protéines consti- tuaient réellement les déterminants $\mathrm{Sm}$ et RNP. Deux polypeptides de poids moléculaire $68 \mathrm{~K}$ et/ou de 33 $\mathrm{K}$ ou $22 \mathrm{~K}$ daltons selon les auteurs sont révélés par les anti-RNP [8]. Des anticorps monoclonaux antiRNP ont aussi détecté ces protéines [9]. D'autres protéines ne sont reconnues que par certains sérums.

Trois protéines présentent des déterminants antigéniques correspondant à l'antigène $\mathrm{Sm}$, leur poids moléculaire est respectivement 13 $\mathrm{K}, 26 \mathrm{~K}$ et $27 \mathrm{~K}$ daltons [8]. L'association de ces antigènes avec les HnRNP [10] et la matrice nucléaire [11] a été démontrée. Ces antigènes sont très conservés puisqu'ils existent chez la drosophile. Toutefois des différences dans le poids moléculaire des protéines ont été notés.

Antigène La (SS-B) et Ro (SS-A) Les antigènes $\mathrm{La}$ et Ro quoique sensibles à la trypsine et résistants à la RNase sont aussi des ribonucléoprotéines (RNP). L'immunopréci-

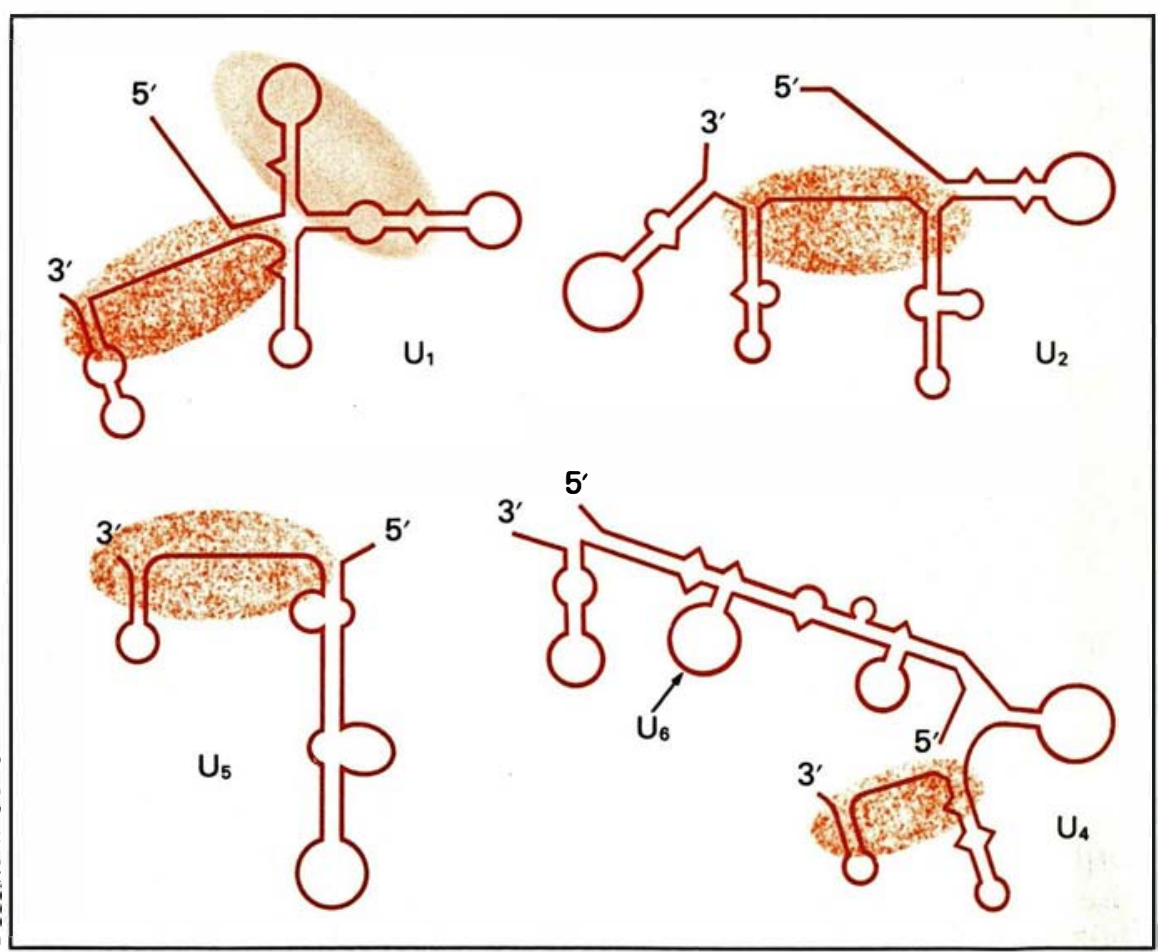

Figure I. Représentation schématique de la structure secondaire des $s n R N A$ avec les zones d'interaction des protéines conférant l'antigénicité.

Les snRN $A$ ne sont pas antigéniques par eux-mêmes. Ce sont des protéines fortement associées qui conferent la spécificité antigénique. Les protéines $S_{m}$ sont retrouvées sur les snRN $A U_{I}, U_{2}, U_{4}, U_{5}$, elles reconnaissent une structure en $A R N$ simple brin comprenant la séquence $A(U) n G[7]$. Les protéines $R N P$ ne sont associées qu'au snRNA $U_{I}$ et protègent la première moitiè de l' $A R N$, à l'exclusion de 'extrémité 5', d'une digestion nucléasique [20]. 


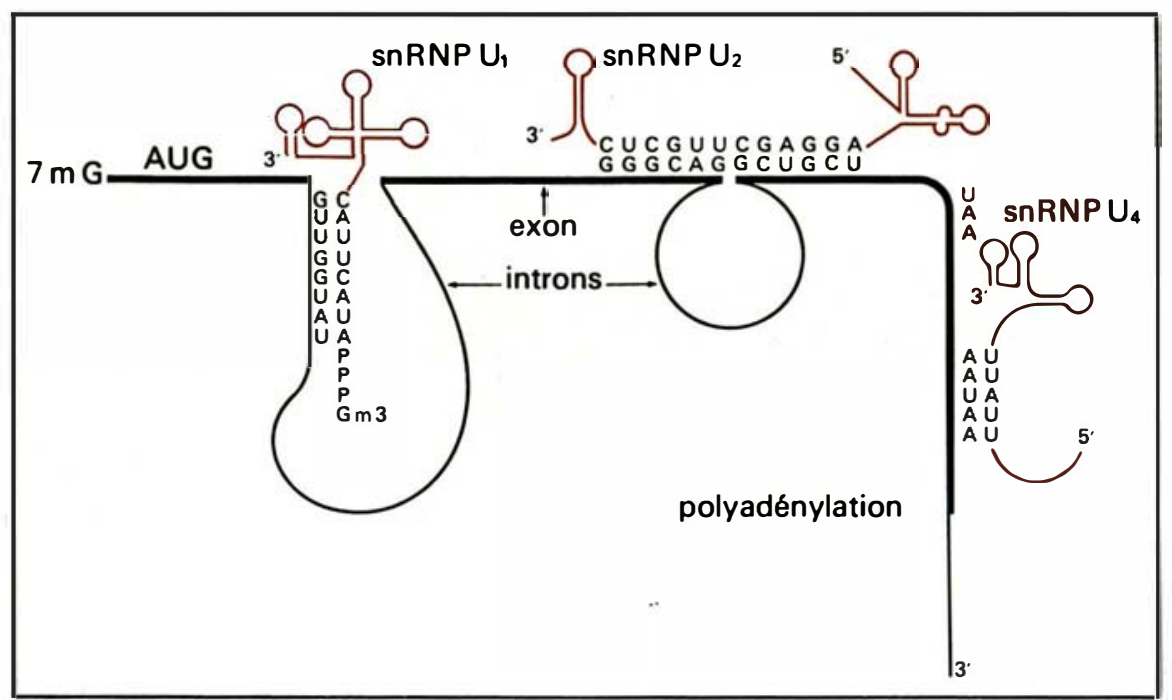

Figure 2. Représentation schématique de linteraction des snRNP avec l $A R N$ pre-messager (pre-mRNA).

Les snRNP, reconnues par les autoanticorps, sont impliquées dans la maturation des messagers. La reconnaissance des sites d'interaction est réalisée, au moins en partie, par un appariement de bases complémentaires. La snRNP UI reconnait une séquence consensus présente aux extrémités de lintron. La représentation est celle de Keller modifiée [25]. Un rôle similaire a été proposé pour la snRNP U2. Toutefois lappariement des bases ne serait réalisé que sur certains messagers puisque la SnRNP U2 reconnait une séquence codante [26]. L'ARN polymérase II transcrit le gène au delà des séquences retrouvées dans le $m R N A$. La maturation consiste donc en lélimination de lextrémité $3^{\prime}$ et en l'addition d'une séquence poly $A$. La snRNP $U_{4}$ permet la reconnaissance du site de polyadénylation par interaction avec la séquence consensus AAUAA. Le schéma est inspiré du modèle de Berget [27].

pitation d'extraits cellulaires a révélé la nature des RNAs. Plusieurs ARN de petite taille ont été identifiés. Ce sont tous des ARN transcrits par l'ARN-polymerase III. Ils sont donc tous terminés par un triphosphate à l'extrémité 5 '. L'antigène $\mathrm{La}$ contient soit des ARN cellulaires $(4,5 \mathrm{~S}$, pre-RNA 5S, RNA 7-2S, pre tRNA, RNA Y) [12] soit des ARN viraux (VA, $\mathrm{VA}_{\text {II }}, \mathrm{EBER}_{1}$ et EBER ${ }_{\text {II }}$ ) [12]. Par contre l'antigène Ro ne semble contenir que les ARN du type Y. Ces ARN ont d'ailleurs été découverts grâce aux antigènes Ro [12] car ils existent en faible quantité (environ 200000 copies par cellule), leur gène a été cloné [13]. La majorité des ARN Y semblent se trouver dans le cytoplasme alors que les autres sont surtout nucléaires [12]. L'antigène Ro présente aussi les déterminants $\mathrm{La}$ et serait donc une sous-classe de ce dernier [14]. Deux protéines ont été trouvées associées à l'antigène Ro par Francœur et Mathews [15], leur poids molécu- essentielle : ils sont très conservés au cours de l'évolution, ce qui implique un rôle fondamental dans la physiologie des organismes eucaryotes.

L'étude des anticorps autoimmuns a non seulement permis d'attirer l'attention sur ces RNP et d'étudier leur structure, mais a permis aussi de découvrir ou de préciser leur rôle biologique.

Rôle des snRNP dans la maturation des ARN messagers

Il est clair aujourd'hui que la particule contenant le snRNA $U_{1}$ joue un rôle important dans l'épissage (splicing) des pre-mRNA (précurseur nucléaire des mRNA). Après les hypothèses basées sur une complémentarité entre l'extrémité 5 ' du snRNA $U_{1}$ et les séquences consensus des régions frontières entre les exons et les introns [21], l'inhibition par les autoanticorps de l'épissage in vitro [22] et son activation par l'addition de snRNP [23] démontrent l'implication directe des snRNP dans les mécanismes de maturation des pre-mRNA.

Enfin Padgett et al. [24] ont pu montrer que les sérums anti-Sm et anti-RNP inhibent l'épissage alors que le sérum anti-snRNP $U_{2}$ est sans effet. Il ne fait donc pas de doute que la snRNP $U_{1}$ joue un rôle important dans l'épissage mais le mécanisme exact est plus complexe que celui proposé initialement par Lerner et Steitz [21] où le snRNA $\mathrm{U}_{1}$ faisait simplement un pont moléculaire entre les deux exons pour permettre l'élimination de l'intron. En effet, plusieurs travaux récents ont démontré que l'épissage passait par une forme en "lasso " (lariat) qui implique une reconnaissance à l'intérieur de l'intron [25]. Le rôle des autres $\operatorname{snRNP}\left(\mathrm{U}_{2}, \mathrm{U}_{4}\right.$, $\mathrm{U}_{5}$ et $\mathrm{U}_{6}$ ) n'est pas établi de façon définitive mais des hypothèses basées (comme ce fut le cas initialement pour la snRNP $U_{1}$ ) sur des homologies de séquence, leur attribuent un rôle dans différentes étapes de la maturation des ARN prémessagers (figure 2).

Le snRNA $U_{2}$ a été impliqué dans l'épissage de certains pré-mRNA [26]. Le snRNA $U_{4}$ possède une séquence nucléotidique complémentaire aux régions servant de 


\section{REFERENCES}

19. Rosa MD, Hendrick JP, Lerner MR, Steitz JA, Reichlin M. A mammalian tRNA ${ }^{\text {his }}$ containing antigen is recognized by the polymyositis-specific antibody anti Jo-I. Nucleic Acids Res 1983; 11 : 853-70.

20. Brunel C, Sri-Widada J, Jeanteur P. SnRNPs and ScRNPs in eucaryotic cells. Prog Mol Subcell Biol 1985; 9: sous presse.

21. Lerner MR, Boyle J, Mount S, Wolin S, Steitz JA. Are snRNPs involved in splicing? Nature 1 980; 283: 220-4.

22. Yang VW, Lerner MR, Steitz JA, Flint SJ. A small nuclear ribonucleoprotein is required for splicing of adenoviral early RNA sequences. Proc Natl Acad Sci USA 1 981; 78: 137 1-5.

23. Hernandez N, Keller W. Splicing of in vitro synthesized messenger RNA precursors in HeLa cell extracts. Cell 1983; 35: 89-99.

24. Padgett RA, Mount SM, Steitz JA, Sharp PA. Splicing of messenger RNA precursors is inhibited by antisera to small nuclear ribonucleoprotein. Cell 1983; 35: 101-7.

25. Keller W. The RNA Lariat: a new ring to the splicing of mRNA precursors. Cell 1984; 39: 423-5.

26. Oshima $Y$, Itoh $M$, Okada $N$, Miyata $T$. Novel models for RNA splicing that involve a small nuclear RNA. Proc Natl Acad Sci USA I $981 ; 78: 4471-4$

27. Berget $\mathrm{SM}$. Are $\mathrm{U}_{4}$ small nuclear ribonucleoproteins involved in polyadenylation? Nature 1984;309: 1 79-82.

28. Moore CL, Sharp PA. Site-specific polyadenylation in a cell-free reaction. Cell $1984 ; 36$ : $581-91$.

29. Gottesf eld JM, Andrews DL, Hoch SO. Association of an RNA polymerase III ranscription factor with a ribonucleoprotein complex recognized by autoimmune sera. Nucleic Acids Res 1984; 1 2:31 85-200.

30. Reichel P, Merrick WC, Scekerka J, Mathews $M$. Regulation of a protein synthesis initiation factor by adenovirus-associated RNA. Nature 1981; 31 3:196-200.

3I. Habets W, De Roois D, Hoet M, Van de Putte L, Van Venrooij W. Quantitation of anti-RNP and anti-Sm antibodies in MCTD and SLE patients by immunoblotting. Clin Exp Immunol 1985;59:457-66.

32. Lindgren V, Ares M, Weine A, Francke U. Human gene for $U_{2}$ small nuclear RNA map to a major adeno 12 modification site on chromosome 17. Nature 1985; 3 I 4: I 1 5-6.

\section{TIRES A PART}

J.P. Liautard : Inserm U 249 et CRBM du Cnrs Route de Mende, B.P. 505 I, 34033 Montpellier acedex. signal à la polyadénylation [27]. De plus, Moore et Sharp [28] ont montré que les anti-Sm inhibent la polyadénylation dans un système acellulaire. Récemment, plusieurs groupes ont mis en évidence l'association des particules $U_{4}$ et $U_{6}$ et, à cette occasion, un rôle regulateur de la polyadénylation par la snRNP $U_{6}$ a été évoquée [20].

Rôles de l'antigène La dans la transcription

Steitz et coll. [16] ont montré que les extraits de transcription acellulaire épuisés en antigène La ne synthétisent plus les petits $A R N$ viraux EBER I et EBER II. De plus, les anticorps anti-La précipitent l'ADN du gène VA cloné, dans un système de transcription in vitro, impliquant une association $\mathrm{ADN}$, ARN-VA, protéine La. Ils ont donc considéré que la protéine La était un facteur de transcription de l'ARN polymérase III. De façon analogue Gottesfeld et coll. [29] ont montré que les anticorps anti-La inhibaient la transcription in vitro des gènes $5 S$, tRNÄmet et $V_{\mathrm{I}}$. Cette inhibition peut être levée par l'addition d'une protéine de $64 \mathrm{~K}$ daltons présente sur la RNP-La, différente de l'antigène principal qui est une protéine de $50 \mathrm{~K}$ daltons. Il faut noter enfin qu'un rôle dans la synthèse des protéines a été proposé pour $\mathrm{VA}_{1}$ [30].

\section{Derniers progrès biochimiques}

Il apparaît désormais de façon évidente que l'éclairage apporté par les techniques biochimiques sur la caractérisation des anticorps antiENA vont être dans les années à venir source de progrès diagnostique considérables. Les deux approches que sont la caractérisation des snRNA précipités et les protéines reconnues en " immunoblot " ont déjà été utilisées par quelques laboratoires avec des résultats très prometteurs.

L'équipe de J. Steitz [9] a déjà pu démontrer l'association fréquente d'anti-RNP et d'anti-Sm dans le lupus érythémateux disséminé (LED) par un simple test de dilution des sérums à tester. En effet, dans le cas où un sérum contient un mélange d'anti-RNP et d'anti-Sm, précipitant comme l'anti-Sm pour les $\operatorname{snRNA} \mathrm{U}_{1}, \mathrm{U}_{2}, \mathrm{U}_{4}, \mathrm{U}_{5}, \mathrm{U}_{6}$, il est possible de trouver une dilution à laquelle seul l'anti-RNP garde son activité et ne précipite donc que le snRNA $U_{1}$.

La précipitation des ARN EBER I et II ou des ARN VA I et VA II pourrait constituer un test très sensible de détection des anticorps anti-La (SS-B); de même, la caractérisation des snRNP de la série $Y$ comme test de détection pour les anticorps anti-Ro (SS-A).

Plus prometteuse encore pourrait être la technique d'" immunoblot" qui permet de caractériser la réaction sérique vis-à-vis d'un ensemble de protéines nucléaires séparées en fonction de leur poids moléculaire. Les premiers résultats publiés par l'équipe de Steitz [9] montrent en effet qu'il est possible de caractériser la spécificité RNP par une réaction constante et forte sur des protéines $33 \mathrm{~K}, 22 \mathrm{~K}$ et plus faible sur un doublet $26-27 \mathrm{~K}$, une protéine $68 \mathrm{~K}$ étant marquée nettement seulement chez certains malades, pas chez d'autres. Ceci a été confirmé dans un travail non encore publié de notre laboratoire.

Cette approche s'avère être un excellent outil lorsque la faiblesse de la réaction en double immunodiffusion ne permet pas de mettre en évidence l'identité avec un sérum de référence. A l'opposé, la spécificité Sm apparaît comme étant liée à la reconnaissance des protéines $26 \mathrm{~K}$ $27 \mathrm{~K}$ (fortement reconnues cette fois) et surtout $13 \mathrm{~K}$. Dans notre expérience personnelle, une excellente corrélation clinique apparaît alors puisque tous les sérums testés reconnaissant la $13 \mathrm{~K}$ provenaient de patients atteints de LED. Comme dans le travail rapporté par Petterson et coll. [9], certains de ces sérums n'avaient donné aucune réaction en double immunodiffusion ce qui indique que l'utilisation de l' immunoblot " permettra de détecter un nombre plus important de malades avec anticorps anti ENA. En comparant avec un sérum de référence, il est possible de proposer un test semi-quantitatif par dilution qui porterait simultanément sur toutes les spécificités caractérisées. Récemment, Habet et 
coll. [31] ont proposé une méthode quantitative utilisant "l'immunoblotting ". Cette méthode est 500 fois plus sensible que la double immunodiffusion. On attend aussi beaucoup des anticorps monoclonaux qui pourront permettre le dosage par compétition des antigènes (Radioimmunoessais).

Un gros travail de biologie médicale s'ouvre à présent; nul doute qu'il constituera un progrès décisif dans le diagnostic, la compréhension et la surveillance des connectivites autoimmunes. Le passage en routine pour l'exploration des sérums par " immunoblot " a d'ailleurs déjà débuté dans notre laboratoire (CTS Montpellier) mais un recul clinique est encore nécessaire pour en évaluer l'intérêt définitif.

\section{Étiologie des connectivites}

La théorie virale de l'origine des maladies autoimmunes n'est pas nouvelle, mais sa démonstration reste difficile. Nous noterons les faits qui semblent impliquer le rôle de certains virus transformants dans les connectivites.

Le premier argument est apporté par la présence d'ARN viraux dans les antigènes $\mathrm{La}$ qui sont produits en quantité plus importante par les cellules transformées par EBV que par les cellules non transformées. On pourrait donc supposer qu'une augmentation de la production de ces antigènes aboutirait, peut-être, via leur libération par lyse cellulaire, à l'immunisation des sujets infectés et serait donc à l'origine des Goujerot-Sjogren à anti-La. La même hypothèse pourrait être appliquée aux connectivites caractérisées par les anti-RNP et anti-Sm (LED et CM) et expliquer les résultats de Lindgren et coll. [32] qui viennent de montrer que les gènes pour les petits $A R N U_{1}$ et $U_{2}$ sont précisément ceux qui sont activés par le virus transformant Adeno 12. La production d'un excès d'antigènes pourrait initier la maladie. De même, l'infection par un virus entraîne une augmentation de la synthèse des tRNA et pourrait expliquer l'origine des anti-Jol suggérée par Rosa et coll. [19].

Une autre hypothèse expliquerait l'origine virale de certaines connectivites. Il a été montré que des anticorps monoclonaux anti-ADN peuvent reconnaître des protéines en raison de la similitude des épitopes, quoique les molécules soient très différentes. De la même manière, on pourrait supposer que certains virus présentent des épitopes très proches de ceux des RNP et que les anticorps antiviraux reconnaitraient les RNP. De fait, M. Rucheton et coll. (manuscrit soumis) viennent de démontrer l'apparition d'un taux anormal d'anticorps contre les protéines du rétrovirus MSV-MLV codée par le gène $\mathrm{Gag}$ et leur précurseur dans certaines connectivites mixtes. Ces deux types d'hypothèse ne sont pas contradictoires mais pourraient au contraire expliquer la présence de plusieurs antigènes différents chez le même patient.

Ainsi, associée à d'autres facteurs, tel le déséquilibre du sytème immunitaire, l'immunisation contre des ribonucléoprotéines induites par l'infection virale ou contenant des séquences propres au virus, pourrait jouer un rôle déterminant dans le déclenchement des maladies autoimmunes et leur imprimer certaines caractéristiques symptomatiques

\section{Remerciements}

Nous remercions $S$. Camalon, C. Bernié et $B$. Fory pour leur assistance technique durant l'élaboration du manuscrit.

\section{Summary}

Among the autoimmune disorders, a number of connective tissue diseases are characterized by autoantibodies directed against nuclear RNP containing small RNA. Today the nosology of these diseases is, at least partially, related to the nature of the antigenic RNP involved. We present and discuss these relations. Then, we review the recent works on the structure and the function of the nuclear RNP recognized by these autoantibodies. They are all involved in fundamental functions of the cell like splicing, polyadenylation of messenger RNA or in RNA polymerase III directed transcription. The recent knowledge obtained on the structure of RNP allows development of new methods that could be used to diagnose the autoimmune diseases (i. c. quantitative immunoblotting or monoclonal antibodies). Based on recent results we propose a viral origin of connective tissue diseases. 